\title{
The Prevalence of Hypothalamic-Pituitary-Adrenal Axis Suppression in Children with Persistent Asthma
}

\author{
Merve Sakar ${ }^{1}$, Sebnem Ozdogan (iD ${ }^{1,}{ }^{*}$, Ahmet Ucar $^{1}$, Aysenur Kaya ${ }^{1}$, Sermin Aksoy ${ }^{1}$ and Yildiz \\ Yildirmak ${ }^{1}$ \\ ${ }^{1}$ Sisli Hamidiye Etfal Research and Training Hospital, Istanbul, Turkey \\ "Corresponding author: Pediatric Pulmonology, Sisli Hamidiye Etfal Research and Training Hospital, Istanbul, Turkey. Email: ozdogan65@hotmail.com
}

Received 2019 February 21; Revised 2019 June 10; Accepted 2019 June 30.

\begin{abstract}
Background: Hypothalamic-pituitary-adrenal (HPA) axis suppression is the most important systemic side effect associated with inhaled corticosteroids (ICS) therapy.

Objectives: To evaluate the prevalence and determinants of hypothalamic-pituitary-adrenal (HPA) axis suppression in asthmatic children on ICSs.

Methods: A total of 92 children aged 6 to 18 years, diagnosed with persistent asthma, and receiving regular ICS therapy for a minimum of 3 months, without any systemic steroid therapy within the last 3 months, were enrolled in the study. Clinical and demographic variables were recorded. HPA axis suppression was measured by morning cortisol levels and confirmed by low-dose adrenocorticotropic hormone stimulation test.

Results: Of 92 enrolled patients, 51 (55.4\%) were male. The mean age was $10.1 \pm 2.6$ years. HPA axis suppression was observed in two (2.1\%) patients. The body mass index (BMI) values were significantly lower in the HPA axis suppression group compared to the other enrollees. No relationship was observed between the HPA axis suppression and the ICS dose, duration of therapy or coadministration of long-acting beta-agonists or nasal steroids.

Conclusions: In our study, the prevalence of HPA axis suppression was 2.1\%. Children with persistent asthma who has been treated with regular ICS should be screened for HPA axis suppression.
\end{abstract}

Keywords: Hypothalamic-Pituitary-Adrenal Axis Suppression, Asthma, Children, Low-Dose ACTH Stimulation Test, Inhaled Corticosteroids

\section{Background}

Inhaled corticosteroids (ICSs) are the most effective and most commonly used treatment for asthma $(1,2)$. ICSs have been shown to effectively reduce airway inflammation, control asthma symptoms, improve the quality of life, improve the lung function, reduce airway hyperresponsiveness, alleviate the frequency and severity of attacks, and reduce mortality due to asthma $(1,2)$. Hypothalamicpituitary-adrenal (HPA) axis suppression is the most important systemic side effect associated with ICS therapy, and its mechanism is well described. The incidence of the side effects increases parallel to the increase in the daily doses $(2,3)$. Chronic exposure and high doses might result in HPA axis deficiency, adrenal gland atrophy, and insufficient cortisol production due to adrenal suppression. Patients might present with adrenal failure, as well as nonspecific symptoms such as malaise, headache or abdominal pain or more serious symptoms, such as growth re- tardation, syncope, hypotension, hyponatremia or hypoglycemia $(4,5)$.

\section{Objectives}

This study aims to evaluate the prevalence and risk factors of HPA axis suppression in children aged 6 to 18 years followed-up with the diagnosis of persistent asthma and have been under regular ICS therapy for a minimum of 3 months.

\section{Methods}

\subsection{Study Design and Settings}

The present cross-sectional study was conducted at Sisli Hamidiye Etfal Research and Training Hospital, between June 2015 and December 2015. Informed consent was obtained from the subjects and their guardians. The 
parents and patients were informed about the test to be performed by the same physician.

\subsection{Study Population}

The subject group included pediatric patients (6- to 18years-old), who were being followed-up with the diagnosis of persistent asthma and had been receiving regular inhaled corticosteroid therapy for at least 3 months. Those who had received systemic corticosteroid therapy within the last 3 months, who had intermittent asthma, received irregular inhalation therapy or had missing information in their files, were excluded from the study.

\subsection{Study Protocol}

The flowchart of the study is presented in Figure 1. All of the subjects were evaluated for symptoms and signs of adrenal failure. We asked the enrollees about inappetence or increased appetite, nausea, malaise, morning headaches, failure to gain weight associated with HPA axis suppression; autonomic symptoms pointing to hypoglycemia; abdominal pain, fatigue, weakness, myalgia, growth retardation and other subjective clinical signs associated with suppression. Patients with a minimum of three symptoms were included in the study.

Data were recorded for each patient, including the age, gender, height, weight, age at diagnosis, hospitalization in the last one-year period, ICS therapy (type, dose, duration), and other therapies (long-acting $\beta 2$-agonists or nasal corticosteroids) received. The severity of the asthma was classified as mild, moderate or severe persistent asthma according to the National Asthma Education and Prevention Program (NAEPP) guideline (6). Patients with mild asthma were treated with low ICS daily dose, moderate asthma with medium daily dose and severe asthma with medium to high daily dose as per NAEPP guideline (6). ICS was given as metered dose inhaler (MDI), whereas ICS + LABA were given as dry powder inhaler to 12 years and older. Total IgE (T. IgE), inhalant panel, allergy skin test, and pulmonary function test (PFT) were obtained from the patient files. The forced expiratory volume at 1 second to forced vital capacity (FEV1/FVC) ratio lower than $80 \%$ were considered as a bronchoconstrictive change (7).

\subsection{Cortisol Test}

The metyrapone and insulin tolerance tests are considered the gold standards to evaluate HPA axis function in asthmatic patients (8). In the literature, studies conducted using the metyrapone test have revealed the prevalence of HPA axis dysfunction as 65\% and HPA axis suppression as $16 \%$ (9). These rates are suggested to be even higher in asthma patients who receive concomitant ICS and nasal steroid (NS) therapy (10). However, since metyrapone is not licensed in some countries, including Turkey, testing is limited. On the other hand, the insulin tolerance test is not preferred as its safety in children has not been established (4).

The ACTH stimulation test has two variants, the standard $(250 \mu \mathrm{g})$ and low-dose $(1 \mu \mathrm{g})$ options (4). The low-dose ACTH stimulation uses a physiological dose and is a safe and convenient method (11). Moreover, compared to the standard-dose ACTH test, the low-dose ACTH stimulation is a more reliable test, with $100 \%$ specificity and $89 \%$ sensitivity $(12,13)$. The study subjects were referred to the Pediatric Endocrine Outpatient Clinic. After being evaluated by a pediatric endocrine specialist, subjects were scanned for baseline fasting blood cortisol levels at 08:00 am $(14,15)$. Patients whose cortisol levels were lower than $14.5 \mu \mathrm{g} / \mathrm{dL}$ (400 nmol/L) were given the low-dose ACTH stimulation test between 08:00 and 10:00 am. After the patients' baseline blood samples were collected, $1 \mu \mathrm{g}$ of synthetic ACTH (Synacthen ${ }^{\circledR} 0.25 \mathrm{mg}$, Novartis, Germany) was administered intravenously after reconstitution with $0.9 \%$ saline. The drug was procured from abroad. After the administration of the ACTH, to measure the serum cortisol response, blood samples were collected after 30 and $40 \mathrm{~min}$. HPA axis function was considered normal in patients with any cortisol level above $18 \mu \mathrm{g} / \mathrm{dL}(500 \mathrm{nmol} / \mathrm{L})(4,16,17)$. The demographic characteristics of the patients, the clinical and laboratory features of asthma, ICS dose, duration of treatment, and the LABA and nasal steroid use were compared between the groups of patients with normal HPA function and those with suppressed HPA axis, and the risk factors for these characteristics were analyzed.

\subsection{Statistical Analysis}

Statistical analyses were performed using the SPSS 15.0 for Windows software. For the numerical variables, descriptive statistics were given as the mean, standard deviation, minimum, and maximum. Since the condition for normal distribution was not fulfilled, the numerical variables between the two independent groups were compared using the Mann Whitney U-test. The ratios between the groups were compared with the help of the chi-square test. The Monte Carlo simulation was used wherever the conditions were not fulfilled. The alpha statistical level of significance was based on a value of $\mathrm{P}<0.05$.

\section{Results}

Ninety-two patients with persistent asthma, among which 51(55.4\%) were male, were included in the study. HPA axis suppression was observed in $2(2.1 \%)$ of the 92 patients 


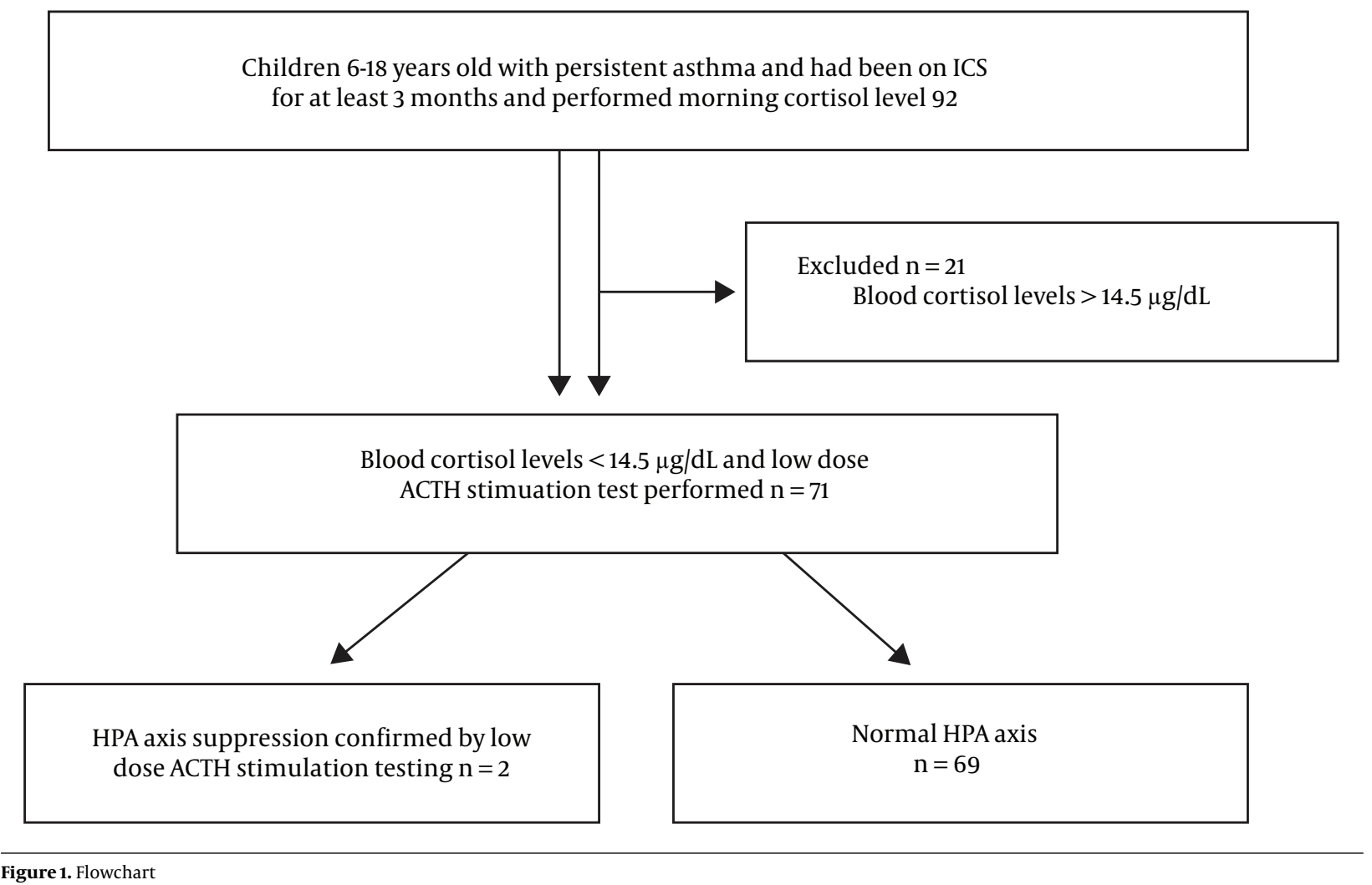

Figure 1. Flowchart

with low morning serum cortisol level. The demographic characteristics of the patients with and without HPA axis suppression are presented in Table 1 . No statistically significant difference in terms of age, gender, weight, and height were observed between the groups $(\mathrm{P}>0.05)$. The BMI values of the patients with HPA axis suppression, as measured by the low-dose ACTH test, were significantly lower than those without HPA axis suppression $(\mathrm{P}=0.034)$.

The clinical features of asthma and the laboratory test results of the patients with and without HPA axis suppression, as measured by the low-dose ACTH test, are shown in Table 2. Both groups were comparable in terms of the age at the diagnosis of asthma, the severity of asthma, and the number of hospitalizations due to acute asthma attacks in the last 1-year period. No statistically significant difference was observed in terms of the laboratory features of asthma including the T. IgE levels, elevated age-related T. IgE, positive inhalant panel, positive skin test, and PFT ( $\mathrm{P}>0.05)$. When the characteristics of the asthma treatment were compared between the patients with and without HPA axis suppression, as measured by the low-dose ACTH test, no statistically significant difference was found in terms of the nasal steroid use, duration of ICS therapy, ICS dose, ICS type, cumulative dose or blood cortisol level ( $\mathrm{P}>0.05)$.
Table 1. The Demographic Characteristics of the Patients with and Without Hypothalamic-Pituitary-Adrenal (HPA) Axis Suppression Confirmed by Low Dose Adrenocorticotropic Hormone (ACTH) Stimulation Testing ${ }^{\mathrm{a}}$

\begin{tabular}{|c|c|c|c|}
\hline & $\begin{array}{c}\text { Normal HPA Axis, } \\
\quad \mathrm{N}=69(92.2 \%)\end{array}$ & $\begin{array}{c}\text { HPA Axis } \\
\text { Suppression, } N= \\
2,(2.8 \%)\end{array}$ & P Value \\
\hline Age & $10.2 \pm 2.6$ & $8.5 \pm 0.7$ & 0.342 \\
\hline Gender & & & 1.000 \\
\hline Female & $32(46.4)$ & $1(50.0)$ & \\
\hline Male & $37(53.6)$ & $1(50.0)$ & \\
\hline Weight, kg & $43.3 \pm 14.8$ & $30.3 \pm 8.1$ & 0.144 \\
\hline Height, cm & $142.1 \pm 16.4$ & $140.0 \pm 14.1$ & 0.903 \\
\hline $\mathbf{B M I}^{\mathbf{b}}$ & $21.0 \pm 4.2$ & $15.3 \pm 1.1$ & 0.034 \\
\hline \multicolumn{4}{|l|}{ BMI } \\
\hline Normal & $30(43.5)$ & $2(100)$ & 0.695 \\
\hline $\begin{array}{l}\text { Under- } \\
\text { weight }\end{array}$ & $1(1.4)$ & $0(0.0)$ & \\
\hline Overweight & $14(20.3)$ & $0(0.0)$ & \\
\hline Obese & $24(34.8)$ & $0(0.0)$ & \\
\hline
\end{tabular}

Abbreviation: BMI, body mass index

${ }^{a}$ Values are expressed as mean \pm SD or No. (\%).

${ }^{\mathrm{b}}$ Mann Whitney U test; chi square

The clinical characteristics of the two patients with 


\begin{tabular}{|c|c|c|c|}
\hline & Normal ACTH Axis, $\mathrm{N}=69(92.2 \%)$ & ACTH Axis Suppression, $\mathrm{N}=2(2.8 \%)$ & P Value $^{\text {b }}$ \\
\hline Age at diagnosis of asthma & $6.3 \pm 3.5$ & $5.0 \pm 1.4$ & 0.565 \\
\hline \multicolumn{4}{|l|}{ Asthma severity } \\
\hline Mild & $33(47.8)$ & $0(0.0)$ & 0.526 \\
\hline Moderate & $34(49.3)$ & $2(100)$ & \\
\hline Severe & $2(2.9)$ & $0(0.0)$ & \\
\hline Hospital admission due to asthma in the last 12 months & $21(30.4)$ & $0(0.0)$ & 1.000 \\
\hline T. IgE levels & $481.0 \pm 609.7$ & $607.0 \pm 834.4$ & 0.941 \\
\hline T. IgE (high) & $43(66.2)$ & $1(50.0)$ & 1.000 \\
\hline Inhalant panel (positive) & $38(63.3)$ & $1(50.0)$ & 1.000 \\
\hline Skin test (positive) & $26(66.7)$ & $1(100)$ & 1.000 \\
\hline PFT (obstructive changes) & $13(18.8)$ & $0(0.0)$ & 1.000 \\
\hline Nasal steroid & $45(65.2)$ & $2(100)$ & 0.546 \\
\hline ICS duration, mo & $11.1 \pm .8$ & $9.0 \pm 0.0$ & 0.643 \\
\hline ICS dose, $\mu$ g/day & $468.0 \pm 122.8$ & $500.0 \pm 0.0$ & 0.609 \\
\hline \multicolumn{4}{|l|}{ ICS type } \\
\hline ICS & $50(72.5)$ & $2(100)$ & 1.000 \\
\hline $\mathrm{ICS}+\mathrm{LABA}$ & $19(27.5)$ & $0(0.0)$ & \\
\hline Blood cortisol levels, mean $\pm \mathrm{SD} /$ min-max & 9.22 .7 & $7.9 \pm 7.0$ & 0.879 \\
\hline Cumulative dose, $\mathrm{mg}$ & $46.3 \pm 140.7$ & $121.5 \pm 6.4$ & 0.821 \\
\hline
\end{tabular}

Abbreviations: ICS, inhaled corticosteroids; LABA, long acting $\beta 2$ agonist; PFT, pulmonary function test

${ }^{\mathrm{a}}$ Values are expressed as mean \pm SD or No. (\%).

${ }^{\mathrm{b}}$ Mann Whitney U test, chi square test

HPA axis suppression, as measured by the low-dose ACTH test, are shown in Table 3.

\section{Discussion}

In this study, we have examined the prevalence of HPA axis suppression as measured by the low-dose ACTH stimulation test in children aged 6 to 18 years, followed-up with the diagnosis of persistent asthma, and receiving regular ICS therapy for a minimum of 3 months. According to our results, the rate of the HPA axis suppression is $2.1 \%$. This ratio is considerably lower than the $7.7 \%-65.1 \%$ prevalence reported in the literature, although this varies depending on the test method $(7,9,12,17-21)$. When we compared the patients with and without HPA axis suppression in terms of the demographics and the laboratory and clinical characteristics, we observed no statistically significant differences between the groups, except for the BMI values. However, it should be noted that our statistical comparison is based on only two patients, in which we have observed HPA axis suppression. In a similar study conducted at the Hacettepe Faculty of Medicine by Cavkaytar et al. (18), the prevalence of the HPA axis suppression was 7.7\%. This study focused on the HPA axis suppression rate and had retrospectively evaluated 91 patients with a reported treatment compliance of $80 \%$ to $90 \%$, which might explain the higher prevalence observed. In our opinion, since our subject group comprised patients from lower socioeconomic groups presenting to our hospital, the lower ratio of HPA axis suppression we observed might be associated with an inaccurate inhaler technique, popular misconceptions about the therapy among patients (e.g., that the drugs cause short stature, trigger asthma), and least likely noncompliance since the drug use was queried by two physicians. Patients who had received systemic steroid therapy due to asthma attacks or for asthma control within the last 3 months were excluded from the study. On the other hand, the study by Cavkaytar et al. (18) included patients who had received systemic steroids due to asthma attacks within the last 3 months and the rate of systemic steroid use due to asthma attacks was higher in the group with HPA axis suppression. A large-scale, case-control study conducted by Mortimer et al. (22) has shown that exposure to oral corticosteroids increases the risk of adrenal failure 
Table 3. Clinical Features of Children with Hypothalamic-Pituitary-Adrenal (HPA) Axis Suppression

\begin{tabular}{lcc}
\hline & Case 1 & Case 2 \\
\hline Age, $\mathbf{y}$ & 9 & 8 \\
\hline Gender & $\mathrm{K}$ & $\mathrm{E}$ \\
\hline Asthma severity & Moderate persistent & Moderate persistent \\
\hline BMI, kg/m & $\mathbf{2}$ & 14.5 \\
\hline T. IgE, high & 16 & yes \\
\hline Inhalant panel & no & positive \\
\hline Skin test & negative & positive \\
\hline PFT & negative & normal \\
\hline ICS dose, $\mu /$ day & normal & 500 \\
\hline ICS duration, months & 500 & 9 \\
\hline $\begin{array}{l}\text { Blood cortisol levels, } \\
\text { nmol/L }\end{array}$ & 9 & 2.9 \\
\hline ICS & 12.8 & Fluticasone \\
\hline Nasal steroid & Fluticasone & propionate \\
\hline $\begin{array}{l}\text { Abbreviations: BMI, body } \\
\text { monary function test }\end{array}$ & yes & yes \\
\hline
\end{tabular}

in patients treated with ICSs. We believe that the exclusion of the patients treated with systemic steroids from our study was a factor leading to lower rates of HPA axis suppression. The best test for evaluating HPA axis function is a controversial topic. In the study conducted by Cavkaytar et al. (18), similarly to our study, the low-dose ACTH test was performed in patients with low morning cortisol levels. However, these authors have determined the limit for peak cortisol level as $19.8 \mu \mathrm{g} / \mathrm{dL}$. In our study, the limit for peak cortisol level was $18 \mu \mathrm{g} / \mathrm{dL}$. In the literature, peak cortisol levels $<18 \mu \mathrm{g} / \mathrm{dL}$ are reported to have $90 \%$ sensitivity and specificity in the diagnosis of adrenal suppression (4). We have chosen our limit based on this finding.

There are studies suggesting that long-term nasal steroid therapy used in the treatment of allergic rhinitis might increase HPA axis suppression when combined with ICSs $(11,12)$. While the ICSs enter the pulmonary circulation after being absorbed from the lungs, nasal steroids directly enter the systemic circulation through the nasal mucosa and lead to stronger systemic effects than the ICSs. This might explain the suppressive effects of nasal steroids. A study conducted by Zollner et al. (10) reported an HPA axis suppression rate of $35 \%$ and suggested that the use of nasal steroids increases the risk of HPA axis suppression. In another study conducted by Zollner et al. (9), a significant correlation was observed between nasal steroids and HPA axis suppression. Also, in our study, the two patients with HPA axis suppression were using nasal steroid therapy.
In the literature, low BMI values are reported among the risk factors of adrenal suppression (14). In our study, the BMI values of the patients who developed HPA suppression were significantly lower than the group with normal HPA axis although this finding depends on only 2 patients. In a study conducted by Zollner et al. (9), the prevalence of HPA axis suppression was lower among patients with higher BMI values. Thus, higher BMI is thought to have a protective effect against HPA axis suppression.

Adrenal failure is one of the acute and life-threatening emergencies faced by physicians. In the literature, there are case reports on adrenal crises that developed secondary to ICS therapy $(23,24)$. In a case series published by Patel et al. (24) comprising eight patients who developed symptomatic adrenal failure due to ICS therapy, two patients were admitted with acute conditions due to hypoglycemia. Drake et al. (25) reported symptomatic hypoglycemia without cushingoid appearance due to adrenal failure in four patients who were receiving high-dose fluticasone propionate therapy ( $>500 \mu \mathrm{g} /$ day). In a survey study from the literature, among 33 patients (28 children, 5 adults) admitted with an acute adrenal crisis, of which 23 were comatose and had convulsions due to acute hypoglycemia, $11 \%$ could not be diagnosed at the first presentation (23). Therefore, pediatricians should be vigilant in case of children with asthma receiving ICS therapy and consider adrenal failure in patients with impaired consciousness, abnormal behavior or autonomic symptoms suggesting hypoglycemia. In our study, none of the patients with HPA axis suppression developed cushingoid appearance or an adrenal crisis. With its many proven benefits on morbidity and mortality in children and adults, ICS therapy forms the basis of asthma therapy and continues to play a critical role in the treatment of childhood asthma. Although systemic side effects due to ICS (e.g., adrenal suppression) usually occur at high doses and through longterm therapy, such effects have also been reported, though rarely, with low- to moderate-dose ICS during short-term treatment (5). Increased awareness, early identification of patients under risk, regular follow-up of the patients to determine the minimum effective ICS dose to control the condition, and reduction of the dose with disease control, are required to reduce the risk of adrenal suppression. Also, selecting the ICS therapy that will cause minimum systemic effects and revision of the therapy before increasing the dose in children with a low level of response might also be helpful $(4,20)$.

In conclusion, we have observed a $2.1 \%$ prevalence of HPA axis suppression in children with asthma who were being followed-up with the diagnosis of persistent asthma and receiving regular ICS therapy. All children in the present study were on medium to low dose of ICS. Children 
with persistent asthma who has been treated with regular ICS should be screened for HPA axis suppression.

The limitations of our study include the low number of subjects, low sensitivity of the measurement of the morning cortisol levels and the inability of normal values to rule out HPA axis suppression, low number of patients with severe asthma, as well as the unavailability of metyrapone, which has been established as the gold standard dynamic test to evaluate HPA axis function, in our country. Another limitation is the study design in which the cross-sectional study does not allow for follow up or repeating the tests.

\section{Footnotes}

Authors' Contribution: Sebnem Ozdogan, Ahmet Ucar, Aysenur Kaya conceived, designed and did editing of manuscript and critical review. Merve Sakar did data collection and manuscript writing. Sebnem Ozdogan did data collection. Yildiz Yildirmak did manuscript review.

Conflict of Interests: It is not declared by the authors.

Ethical Approval: Ethics committee approval was received for this study from the Ethics Committee of Sisli Hamidiye Etfal Research and Training Hospital.

Financial Disclosure: The authors declared no financial disclosure.

Funding/Support: The authors declared no funding/support.

Patient Consent: The written consent of the patients and their parents was obtained who participated in this study.

\section{References}

1. Global Initiative for Asthma. Global strategy for asthma management and prevention. 2017, [cited 20 May 2017]. Available from: http://www.ginasthma.org/2017-gina-report-global-strategy-forasthma-management-and-prevention.

2. Lougheed MD, Lemiere C, Ducharme FM, Licskai C, Dell SD, Rowe BH, et al. Canadian Thoracic Society 2012 guideline update: diagnosis and management of asthma in preschoolers, children and adults. Can Respir J. 2012;19(2):127-64. doi: 10.1155/2012/635624. [PubMed: 22536582]. [PubMed Central: PMC3373283].

3. Heffler E, Madeira LNG, Ferrando M, Puggioni F, Racca F, Malvezzi L, et al. Inhaled corticosteroids safety and adverse effects in patients with asthma.J Allergy Clin Immunol Pract. 2018;6(3):776-81. doi: 10.1016/j.jaip.2018.01.025. [PubMed: 29408385].

4. Ahmet A, Kim H, Spier S. Adrenal suppression: A practical guide to the screening and management of this under-recognized complication of inhaled corticosteroid therapy. Allergy Asthma Clin Immunol. 2011;7:13. doi: 10.1186/1710-1492-7-13. [PubMed: 21867553]. [PubMed Central: PMC3177893].

5. Issa-El-Khoury K, Kim H, Chan ES, Vander Leek T, Noya F. CSACI position statement: Systemic effect of inhaled corticosteroids on adrenal suppression in the management of pediatric asthma. Allergy Asthma Clin Immunol. 2015;11(1):9. doi: 10.1186/s13223-015-0075-z. [PubMed: 25802532]. [PubMed Central: PMC4369840].
6. NHLBI; NIH. Guidelines for the diagnosis and management of asthma (EPR-3). 2007, [cited May 12, 2015]. Available from: http://www.nhlbi nih.gov/health-pro/guidelines/current/asthma-guidelines.

7. American Thoracic Society. Standardization of spirometry, 1994 update. Am J Respir Crit Care Med. 1995;152(3):1107-36. doi: 10.1164/ajrccm.152.3.7663792. [PubMed: 7663792].

8. Zollner EW. Hypothalamic-pituitary-adrenal axis suppression in asthmatic children on inhaled corticosteroids: Part 1. Which test should be used? Pediatr Allergy Immunol. 2007;18(5):401-9. doi: 10.1111/j.13993038.2007.00540.x. [PubMed: 17561933].

9. Zollner EW, Lombard CJ, Galal U, Hough FS, Irusen EM, Weinberg E. Hypothalamic-pituitary-adrenal axis suppression in asthmatic school children. Pediatrics. 2012;130(6):e1512-9. doi: 10.1542/peds.20121147. [PubMed: 23147980].

10. Zollner EW, Lombard C, Galal U, Hough S, Irusen E, Weinberg E. Hypothalamic-pituitary-adrenal axis suppression in asthmatic children on inhaled and nasal corticosteroids-more common than expected? J Pediatr Endocrinol Metab. 2011;24(7-8):529-34. doi: 10.1515/jpem.2011.198. [PubMed: 21932593].

11. Kazlauskaite R, Evans AT, Villabona CV, Abdu TA, Ambrosi B, Atkinson $\mathrm{AB}$, et al. Corticotropin tests for hypothalamic-pituitary- adrenal insufficiency: A metaanalysis. J Clin Endocrinol Metab. 2008;93(11):424553. doi: 10.1210/jc.2008-0710. [PubMed: 18697868].

12. Ozbek OY, Turktas I, Bakirtas A, Bideci A. Evaluation of hypothalamicpituitary-adrenal axis suppression by low-dose (0.5 microg) and standard-dose (250 microg) adrenocorticotropic hormone (ACTH) tests in asthmatic children treated with inhaled corticosteroid. J Pediatr Endocrinol Metab. 2006;19(8):1015-23. doi: 10.1515/jpem.2006.19.8.1015. [PubMed: 16995587].

13. Gonc EN, Kandemir N, Kinik ST. Significance of low-dose and standard-dose ACTH tests compared to overnight metyrapone test in the diagnosis of adrenal insufficiency in childhood. Horm Res. 2003;60(4):191-7. doi: 10.1159/000073232. [PubMed: 14530608].

14. Woods CP, Argese N, Chapman M, Boot C, Webster R, Dabhi V, et al. Adrenal suppression in patients taking inhaled glucocorticoids is highly prevalent and management can be guided by morning cortisol. Eur J Endocrinol. 2015;173(5):633-42. doi: 10.1530/EJE-15-0608. [PubMed: 26294794]. [PubMed Central: PMC4588051].

15. Zollner EW. Asthma treatment in children: A guide to screening for and management of hypothalamic-pituitaryadrenal axis suppression. S Afr Med J. 2019;109(5):306-9. doi: 10.7196/SAMJ.2019.v109i5.13932. [PubMed: 31131795].

16. Wade M, Baid S, Calis K, Raff H, Sinaii N, Nieman L. Technical details influence the diagnostic accuracy of the 1 microg ACTH stimulation test. EurJEndocrinol. 2010;162(1):109-13. doi: 10.1530/EJE-09-0746. [PubMed: 19797501]. [PubMed Central: PMC2941345].

17. Sim D, Griffiths A, Armstrong D, Clarke C, Rodda C, Freezer N. Adrenal suppression from high-dose inhaled fluticasone propionate in children with asthma. Eur Respir J. 2003;21(4):633-6. doi: 10.1183/09031936.03.00306302. [PubMed: 12762348].

18. Cavkaytar O, Vuralli D, Arik Yilmaz E, Buyuktiryaki B, Soyer O, Sahiner UM, et al. Evidence of hypothalamic-pituitary-adrenal axis suppression during moderate-to-high-dose inhaled corticosteroid use. Eur J Pediatr. 2015;174(11):1421-31. doi: 10.1007/s00431-015-2610-9. [PubMed: 26255048].

19. Kannisto S, Korppi M, Remes K, Voutilainen R. Adrenal suppression, evaluated by a low dose adrenocorticotropin test, and growth in asthmatic children treated with inhaled steroids. J Clin Endocrinol Metab. 2000;85(2):652-7. doi: 10.1210/jcem.85.2.6336. [PubMed: 10690871].

20. Paton J, Jardine E, McNeill E, Beaton S, Galloway P, Young D, et al. Adrenal responses to low dose synthetic ACTH (Synacthen) in children receiving high dose inhaled fluticasone. Arch Dis Child. 2006;91(10):808-13. doi:10.1136/adc.2005.087247. [PubMed: 16556614]. [PubMed Central: PMC2066000]. 
21. Smith RW, Downey K, Gordon M, Hudak A, Meeder R, Barker S, et al. Prevalence of hypothalamic-pituitary-adrenal axis suppression in children treated for asthma with inhaled corticosteroid. Paediatr Child Health. 2012;17(5):e34-9. doi: 10.1093/pch/17.5.e34. [PubMed: 23633903]. [PubMed Central: PMC3381924].

22. Mortimer KJ, Tata LJ, Smith CJ, West J, Harrison TW, Tattersfield AE, et al. Oral and inhaled corticosteroids and adrenal insufficiency: A casecontrol study. Thorax. 2006;61(5):405-8. doi: 10.1136/thx.2005.052456. [PubMed: 16517576]. [PubMed Central: PMC2111185].

23. Todd GR, Acerini CL, Ross-Russell R, Zahra S, Warner JT, McCance D. Survey of adrenal crisis associated with inhaled corticosteroids in the United Kingdom. Arch Dis Child. 2002;87(6):457-61. doi: 10.1136/adc.87.6.457. [PubMed: 12456538]. [PubMed Central: PMC1755820].

24. Patel L, Wales JK, Kibirige MS, Massarano AA, Couriel JM, Clayton PE. Symptomatic adrenal insufficiency during inhaled corticosteroid treatment. Arch Dis Child. 2001;85(4):330-4. doi: 10.1136/adc.85.4.330. [PubMed: 11567945]. [PubMed Central: PMC1718935].

25. Drake AJ, Howells RJ, Shield JP, Prendiville A, Ward PS, Crowne EC. Symptomatic adrenal insufficiency presenting with hypoglycaemia in children with asthma receiving high dose inhaled fluticasone propionate. BMJ. 2002;324(7345):1081-2. doi: 10.1136/bmj.324.7345.1081. [PubMed: 11991916]. [PubMed Central: PMC1123030]. 